\title{
Identity Verification Through Palm Vein and Crease Texture
}

\author{
Kar-Ann Toh ${ }^{1}$, How-Lung Eng ${ }^{1}$, Yuen-Siong Choo $^{2}$, \\ Yoon-Leon Cha ${ }^{2}$, Wei-Yun Yau ${ }^{1}$, and Kay-Soon Low ${ }^{2}$ \\ 1 Institute for Infocomm Research, \\ 21 Heng Mui Keng Terrace, Singapore 119613 \\ katoh@ieee.org, \{hleng, wyyau\}@i2r.a-star.edu.sg \\ 2 School of Electrical \& Electronic Engineering, \\ Nanyang Technological University, Singapore \\ ekslow@ntu.edu.sg
}

\begin{abstract}
In this paper, an identity verification framework which combines pattern information from the palm-vein and the palm-crease texture is proposed. Main feature of this system is the use of a low cost Near-Infra-Red (NIR) camera instead of the more expensive infra-red thermal camera for palm image capture. Our preliminary experiments show that useful information from palm-vein and palm-crease texture can be effectively extracted for identity verification using a simple setup to contain the camera.
\end{abstract}

Keywords: Biometrics, Multimodal Biometrics, Palm-vein Recognition, Palm-print Recognition and Pattern Classification.

\section{Introduction}

The palm dermatoglyphics (palmprints) [1,2, 3, 4, 5] and hand vascular network (veins from back of hand) 66,7] have increasingly gained research attention recently. Main reason for this growth, perhaps, can be attributed to the nonintrusive nature and the good inter class differentiability offered by these biometrics. The non-intrusive property includes whether it is linked to criminal records. Palm dermatoglyphics and hand vascular network are advantages over fingerprint and face biometrics in this aspect. The good inter class differentiability to distinguish individual identities are seen from more and more empirical evidences over recent years from the above cited works.

In this work, a low-cost NIR camera, rather than a considerably more expensive Infra-Red (IR) or specially designed thermal camera, is used for capturing the palm image. Since the NIR camera is operating within the visible electromagnetic spectrum, the images captured from this device contain certain amount of palm-crease texture information on top of the thermal vein patterns. We shall explore in this paper, a biometric system whereby feature information are extracted from these two spectrums of palm images for multimodal biometric decision fusion. Main contributions of this work include: (i) definition of palm-vein and 
palm-crease biometric features for NIR palm images which are acquired from a single low cost camera system, and (ii) exploration of fusing these palm-vein and palm-crease texture modalities for identity verification.

\section{Proposed Framework}

\subsection{System Overview}

A low cost monochrome NIR CCD camera (JAI CV-M50 IR [8]) was used to capture the frontal palm images. Since the NIR camera was not sensitive enough to detect the IR radiation emitted by the human body (3000 - $14000 \mathrm{~nm})$, an IR light source was used to irradiate the palm [6]. The camera was mounted on a customized rig with the IR source. Each user was asked to rest his/her hand on a rigid platform with palm facing a hollow cutout where the camera was positioned within. Apart from an alignment point for the placement of middle finger, no additional alignment pads were used.

With appropriate processing to extract information from different spectral zones of the NIR CCD camera, a multi-spectrum palm signature could be available. In this work, we focused only on the visible zone and the NIR zone (600 $\mathrm{nm}$ to $1000 \mathrm{~nm}$ ) of the NIR CCD for palm biometric identity authentication. The images were digitized into $768 \times 576$ pixels (spatial resolution offered by the CCD) with a gray-scale resolution of 8 -bit per pixel.

\subsection{A Bimodal Framework for Identity Verification}

Based on the NIR CCD palm images obtained from the above system, we propose in this paper the following pattern features for identity verification.

Palm-vein feature points. To facilitate fast point-based matching, we propose to represent the palm-vein network structure by a set of points extracted from sub-sampling. A reference grid frame with appropriate size is superimposed on the extracted palm-vein structure, and a palm-vein point is defined as interception point between a vein line and the grid. Although for simplicity reason in this preliminary work, only the location information is included, we shall use the orientation and width information for accuracy enhancement in future.

Palm-crease texture. The palm-crease texture provides another source of information for identity verification. Two main approaches are available for this information extraction, namely local crease-line detection and global texture pattern analysis. In this feasibility study, we adopt the global texture pattern approach by means of elementary wavelet analysis.

Decision fusion. The matching outputs from both the above biometrics are fused at measurement level for final decision to determine whether the query is a genuine-user or an imposter. 


\section{Palm Patterns Recognition}

In this section, the processing steps to extract the palm-vein and palm-crease features are briefly introduced since much of the adopted techniques are based on elementary image processing tools. We shall then show in the experiment section that such elementary modalities can be effectively combined to yield a reasonably accurate bimodal system for identity verification.

\subsection{Region of Interest}

For both palm-vein and palm-crease texture, the comparison (matching) of two identities is based on an image region common to all for computational efficiency reason. We call this the Region of Interest (ROI) [3] and it will be applied to both palm-vein and palm-crease texture biometrics for matching purpose.

\subsection{Verification Using Palm-Vein}

Image pre-processing. High pass filtering: Here, we are only interested in the fairly faint vein line patterns obtained from the NIR spectrum. By carefully analyzing the grey scale palm images, Gaussian high pass filtering is performed with the cut-off frequency determined empirically. The Gaussian filter is first applied to the original image (The intensities of the output image is scaled). The filtered image is then passed through a frequency equalization stage to enhance the desired narrow intensity range. Fig. 1(b) shows the outcome of this processing step.

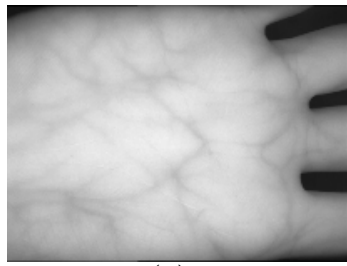

(a)

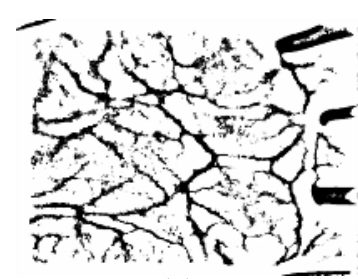

(c)

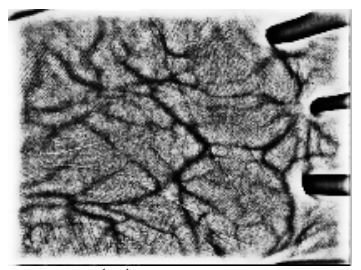

(b)

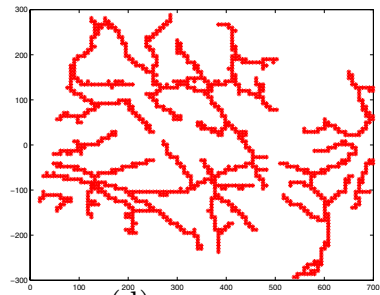

(d)

Fig. 1. Palm-vein processing steps: (a) original image, (b) image after pre-processing, (c) vein-lines feature extraction, and (d) sub-sampled vein lines

Vein-lines feature points extraction. The extraction of vein lines feature begins with a morphological gradient operation, which consists of dilation and 
erosion of an image, to enhance the edges of the vein structure. A morphological gradient operated image is added to the above pre-processed image for further removal of winkles and textures other than the vein. Fig. 1)(c) shows the vein patterns after this processing step.

The positional (movement) and granular noises are further handled by mapping the vein signatures onto a new grid with large grid size. The separation between the grid lines is set to 6 pixels, which means there are 6 pixels in between 2 adjacent grid lines. This grid resolution was selected to provide an adequate representation of the vein network, but at the same time matches the inherent noise associated with the positional information of the vein structure. Fig. 1(d) shows the results of sub-sampling the signatures. The descriptors of the vein structure from the same user produced almost similar diagrams with minimum or no positional noises. In addition, the mapping procedure is also effective in reducing the size of the data which directly improved computational efficiency.

Matching. A template library using the above extracted features within the ROI is constructed for identity verification. The template for each identity is obtained by averaging 5 feature sets from 5 palm samples of the same person. The Correlation Coefficient or the Pearson's correlation coefficient is adopted for matching since it is widely used in statistical analysis, pattern recognition, and image processing.

\subsection{Verification Using Palm-Crease Texture}

Image pre-processing. High-pass filtering: Our interest here is the high frequency palm-crease texture obtained from the visible light spectrum. A Butterworth high-pass filtering is performed to exclude other component frequencies. The cutoff frequency was selected based on empirical observation from applying the filter to images in the database. Fig. 2(b) shows the processed palm image with texture features emphasized.

Palm-crease texture extraction. To extract the features from the ROI subimage (Fig. 2(c)), we use an elementary multi-resolution analysis. At each resolution (scale), multi-resolution analysis decomposes an image into several directions (Horizontal, Vertical and Diagonal). Using the Haar wavelet, three level of decomposition are performed on the image block. At each level of decomposition, the horizontal, vertical and diagonal energy are obtained (Fig. 2(d)). Finally the vector is normalized by the total energy obtained from summing up the elements.

Matching. For identity comparison, a template is first constructed from energy samples of multiple palm images within the ROI. A simple averaging of the multiple energy vectors has been adopted for simplicity and time efficiency reasons. Given two energy representations (test and template resulted from training), the matching module adopts a simple CityBlock (Manhattan or $L_{1}$-norm) distance measure. It is noted that smaller distance denotes better similarity between the two palms in comparison. 


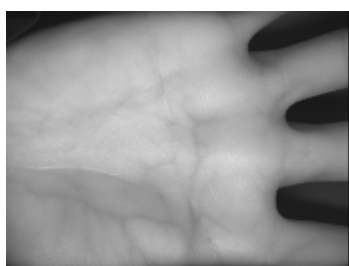

(a)

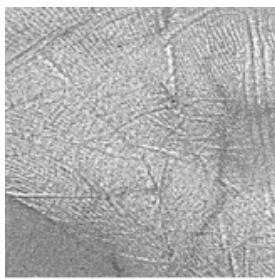

(c)

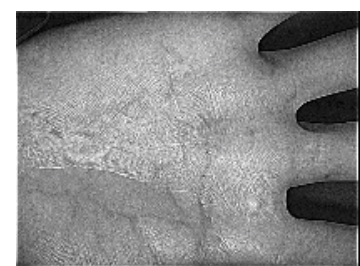

(b)

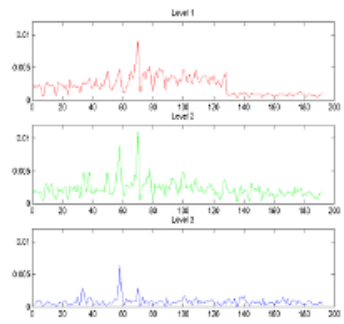

(d)

Fig. 2. Palmprint processing steps: (a) original image, (b) image after high-pass filtering, (c) ROI extraction, and (d) wavelet energy features

\section{Experiments}

\subsection{Database}

A total of 1000 NIR images of the palm are collected as the database for our study - 10 images each from the right and left palm, from 50 different individuals. Since the left and right palms are observed to have significantly different vein patterns, we flip the left palm images to be in similar orientation to those from the right palm and make the assumption that left and right palms constitute different identities. We therefore have 100 identities in total with each identity containing 10 palm image samples.

The data is divided into two sets to accommodate for training and test experiments. We have 5 image samples per individual for each of the training set $(100 \times 5$ images in total $)$ and the test set $(100 \times 5$ images in total $)$. For each training set and each test set, $1000(5 \times 4 \times 100 / 2)$ genuine match scores and $74250(100 \times 99 \times 5 \times 3 / 2)$ imposter match scores are generated for each modality (vein and crease) for performance evaluation. The reason for using only 74250 out of the total possible 123750 $(100 \times 99 \times 5 \times 5 / 2)$ is to have a smaller difference between the imposter and the genuine user data sets. We shall swap between the training set and the test set to create a 2 -fold experiment. Since training results do not indicate predictivity, only the unseen test results will be reported in the sequel.

\subsection{Uni-modal Verifications}

The lower dotted lines in Fig. 3. (a) and Fig. 34(b) show the Receiver Operating Characteristic (ROC) performances for palm-vein and palm-crease-texture 
biometrics for two-fold experiments. The ROC performance of the palm-vein biometric shows significant superiority over that of the palm-crease-texture biometric for the current experimental setting. Main reason is due to better discriminative features obtained from palm-vein line extraction as compared to those generic wavelet energies extracted from the palm-crease-texture.

\subsection{Combining Palm-Vein and Palm-Crease-Texture}

In the next set of experiments, the output from both modalities (vein and crease) are fused at measurement level 9 to form a bimodal verification system. Several classifiers with acclaimed performance (good accuracy and/or fast training speed) are experimented. These classifiers include a simple SUM-rule, Support Vector Machines adopting different kernels (SVM-Linear, SVM-Poly, SVM-RBF) [10] and a Reduced Multivariate polynomial (RM) [1].

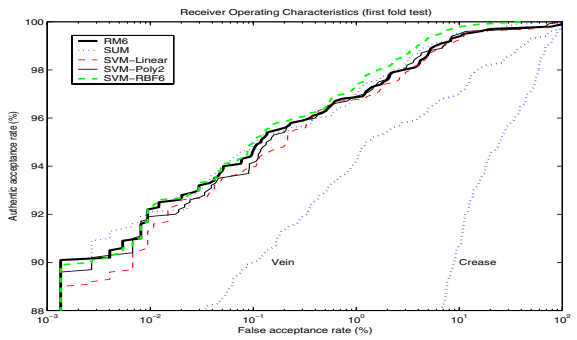

(a)

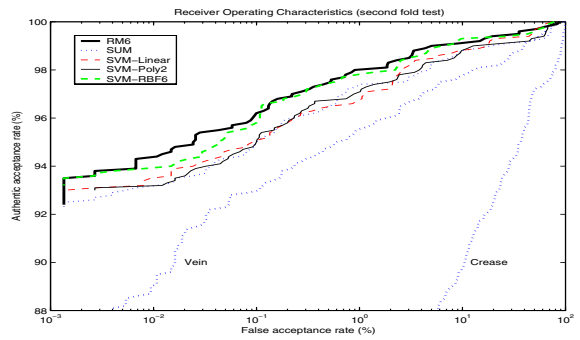

(b)

Fig. 3. ROC plots: (a) First-fold verification test combining palm-vein and palm-creasetexture, (b) Second-fold verification test combining palm-vein and palm-crease-texture

The SUM-rule simply sums up the scores and then divide it by 2 . The SVMPoly was experimented with different polynomial orders ranging from 2 to 8 by validation tests. The best performed order 2 (SVM-Poly2) was presented in Fig. 3-(a) and Fig. 3-(b) respectively for first- and second-fold tests. For SVM-RBF, the scaling parameter Gamma [10] was experimented for values in $[0.1,0.5,1,2,3,4,5,6]$ and the best value was found to be 6 (SVM-RBF6) from validation. The RM was ran from order 2 to order 8 and finally order 6 (RM6) was selected. These selected results for the two-fold tests are shown in Fig. 3-(a) and Fig. 3 -(b).

The results from the plots show that significant accuracy improvement have been achieved by fusing the two modalities for all the compared methods. Among the SVMs, the RBF kernel appears to generalize best for both the two-fold experiments. RM6 appears to achieve good accuracy for most operating ranges. Here, we note the simple SUM-rule achieves remarkably good accuracy for the first-fold test while having a much poorer performance in the second-fold as compared to other classifiers. Here we see that SUM-rule could be a simple and effective method for fusion in this application, but main question remains 
whether the test data fits well with the presumed probabilities for uncorrelated inputs. This finding is congruent with our previous work [12] on decision fusion.

\section{Conclusion}

We proposed to combine the decision measures from two biometrics which were derived from a single palm image obtained from a low cost near-infra-red CCD camera in this paper. The main pattern features adopted for the palm-vein and the palm-crease texture were respectively a set of sub-sampled vein lines network and a set of directional wavelet energies. The two uni-modals are subsequently combined at measurement level to form a bimodal system. Several well acclaimed classifiers (SUM and SVMs) from the literature were experimented comparing with an in-house developed classifier (RM) for decision fusion. Our empirical experiments show that SVM with RBF kernel and RM generalizes best for the two-fold experiments.

\section{References}

1. A. J. Rice, "A quality approach to biometric imaging," in Proceedings of Image Processing for Biometric Measurement IEE Colloquium, 1994, pp. 4/1-4/5.

2. N. Duta, A. K. Jain, and K. V. Mardia, "Matching of palmprints," Pattern Recognition Letters, vol. 23, no. 4, pp. 477-485, 2002.

3. D. Zhang, W.-K. Kong, J. You, and M. Wong, "Online palmprint identification," IEEE Trans. Pattern Analysis and Machine Intelligence, vol. 25, no. 9, pp. 10411050, 2003.

4. L. Zhang and D. Zhang, "Characterization of palmprints by wavelet signatures via directional context modeling," IEEE Trans. Systems, Man and Cybernetics, Part-B, vol. 34, no. 3, pp. 1335-1347, June 2004.

5. A. Kumar, D. C. M. Wong, H. C. Shen, and A. K. Jain, "Personal verification using palmprint and hand geometry bionetric," in Proc. 4th International Conference on Audio- and Video-Based Person Authentication (AVBPA), Guildford, UK, June 2003, pp. 668-678.

6. J. M. Cross and C. L. Smith, "Thermographic imaging of the subcutaneous vascular network of the back of the hand for biometric identification," in IEEE 29th Annual International Carnahan Conference on Security Technology, October 1995, pp. 20-35.

7. C.-L. Lin and K.-C. Fan, "Biometric verification using thermal images of palmdorsa vein patterns," IEEE Trans. Circuits and Systems for Video Technology, vol. 14, no. 2, pp. 199-213, 2004.

8. JAI Camera Solutions, "Near IR industrial CCD camera," in http://www.jai.com/db_datasheet/cvm50irdb.pdf [on-line], 2005, (datasheet).

9. L. Hong and A. Jain, "Integrating faces and fingerprints for person identification," IEEE Trans. Pattern Analysis and Machine Intelligence, vol. 20, no. 12, pp. 12951307, 1998. 
10. J. Ma, Y. Zhao, and S. Ahalt, "OSU SVM classifier matlab toolbox (ver 3.00)," in http://eewww.eng.ohio-state.edu/ maj/osu_svm/, 2002, the Ohio State University.

11. K.-A. Toh, Q.-L. Tran, and D. Srinivasan, "Benchmarking a reduced multivariate polynomial pattern classifier," IEEE Trans. Pattern Analysis and Machine Intelligence, vol. 26, no. 6, pp. 740-755, 2004.

12. K.-A. Toh and W.-Y. Yau, "Combination of hyperbolic functions for multimodal biometrics data fusion," IEEE Trans. Systems, Man and Cybernetics, Part-B, vol. 34, no. 2, pp. 1196-1209, 2004. 